\title{
Factors associated with disability in patients with rheumatoid arthritis with persistent moderate disease activity: a retrospective cohort study
}

Ian C. Scott ${ }^{1,2^{*}}$, Julie Mount ${ }^{3}$, Jane Barry ${ }^{3}$ and Bruce Kirkham ${ }^{4}$

\begin{abstract}
Background: Many patients with rheumatoid arthritis (RA) do not attain remission/low disease activity, remaining in a moderate disease activity state (MDAS) with ongoing disability and impaired quality of life (QoL). If patients in persistent MDAS with poor future outcomes could be prospectively identified, they could arguably be treated more intensively. We evaluated baseline factors predicting function (Health Assessment Questionnaire-Disability Index [HAQ-DI] scores) and QoL (3-level EuroQol-5 dimensions questionnaire [EQ-5D-3L] index scores) at 12 months in patients with RA in persistent MDAS in a real-world setting.

Methods: Patients with persistent MDAS (Disease Activity Score for 28-joint count based on erythrocyte sedimentation rate [DAS28-ESR] 3.2-5.1 on at least two consecutive outpatient appointments over 12 months) were identified retrospectively from Guy's Hospital RA Centre and analysed in two groups: (1) biologic naïve at baseline or (2) receiving/ever received biologics. The baseline timepoint was the second-visit MDAS DAS28-ESR score; the endpoint was the closest visit to 12 months. Linear regression analyses evaluated relationships between baseline variables and (1) 12-month HAQ-DI scores, (2) 12-month rank-transformed EQ-5D-3L index scores, (3) 12-month changes in HAQ-DI scores, and (4) 12-month changes in EQ-5D-3L index scores.

(Continued on next page)
\end{abstract}

\footnotetext{
* Correspondence: i.scott@keele.ac.uk

'Primary Care Centre Versus Arthritis, School of Primary, Community and Social Care, Keele University, Keele, Staffordshire ST5 5BG, UK

${ }^{2}$ Haywood Academic Rheumatology Centre, Haywood Hospital, Midlands

Partnership NHS Foundation Trust, High Lane, Burslem, Staffordshire ST6 7AG,

UK

Full list of author information is available at the end of the article
}

(c) The Author(s). 2020 Open Access This article is licensed under a Creative Commons Attribution 4.0 International License, which permits use, sharing, adaptation, distribution and reproduction in any medium or format, as long as you give appropriate credit to the original author(s) and the source, provide a link to the Creative Commons licence, and indicate if changes were made. The images or other third party material in this article are included in the article's Creative Commons licence, unless indicated otherwise in a credit line to the material. If material is not included in the article's Creative Commons licence and your intended use is not permitted by statutory regulation or exceeds the permitted use, you will need to obtain permission directly from the copyright holder. To view a copy of this licence, visit http://creativecommons.org/licenses/by/4.0/. The Creative Commons Public Domain Dedication waiver (http://creativecommons.org/publicdomain/zero/1.0/) applies to the data made available in this article, unless otherwise stated in a credit line to the data. 


\begin{abstract}
(Continued from previous page)
Results: The analysis included 207 biologic-naïve and 188 biologic-experienced patients. All patients had moderate disability (mean HAQ-DI 1.21 and 1.46) and impaired QoL (mean EQ-5D-3L index scores 0.52 and 0.50). Many reported moderate/severe pain (93 and 96\%) and showed little change in HAQ-DI and EQ-5D-3L index scores over 12 months. In both biologic-naïve and biologic-experienced groups, multivariate analysis revealed a significant association between baseline HAQ-DI scores and endpoint HAQ-DI scores $(\beta=0.67, P<0.001$ and $\beta=0.76, P<$ 0.001 , respectively), 12-month changes in HAQ-DI scores (both $\beta=-0.21, P<0.001$ ), and 12-month EQ-5D-3L index scores $(\beta=-0.57, P<0.001$ and $\beta=-0.29, P=0.004$, respectively). Baseline EQ-5D-3L index scores were significantly associated with 12-month changes in EQ-5D-3L index scores in both groups $(\beta=-0.73, P<0.001$ and $\beta=-0.40$, $P=0.003$, respectively).

Conclusions: Patients with RA in persistent MDAS experience substantial ongoing physical disability, poor QoL, and pain. HAQ-DI is an important predictor of future disability and reduced QoL, supporting current national recommendations to measure HAQ-DI in routine care.
\end{abstract}

Keywords: Rheumatoid arthritis, Moderate disease activity, Disability, Quality of life, Pain

\section{Background}

The established approach to managing patients with rheumatoid arthritis (RA) is treat-to-target (T2T) [1]. This focuses on the gradual up-titration of synthetic diseasemodifying anti-rheumatic drugs (DMARDs), followed by the introduction of biologics and targeted synthetic DMARDs, until the goals of sustained remission or a low disease activity state (LDAS) are attained [2, 3]. In routine clinical practice, remission and LDAS are often defined as the attainment of a Disease Activity Score for 28-joint count based on erythrocyte sedimentation rate (DAS28ESR) of $<2.6$ and $\geq 2.6$ to $<3.2$, respectively.

A key challenge in implementing T2T in routine care is that a substantial minority of patients do not reach remission or LDAS and instead remain in a moderate disease activity state (MDAS), defined as a DAS28-ESR of $\geq 3.2$ to $\leq 5.1$. This was exemplified in two separate observational cohort studies of patients with early RA. First, in the UK-based Early RA Study of 2045 patients with DAS28 recorded at least twice between the first and fifth year of follow-up, $47 \%$ had mean DAS28 scores over time in the MDAS range [4]. Second, in the North America-based CATCH study, which employed group-based trajectory modelling, 10\% of 1586 patients were initially in a high disease activity state (HDAS) and only improved to MDAS over 2 years [5].

There is emerging evidence that patients in persistent MDAS have worse outcomes than those attaining LDAS or remission, exhibiting higher levels of joint damage [4], worse function $[4,6]$, and lower health-related quality of life (HRQoL) [5]. If it were possible to identify patients in persistent MDAS who are most likely to have poor outcomes, then an argument could be made for treating such individuals more intensively. Such treatment could include biologics or targeted synthetic DMARDs, which in the UK are currently restricted to patients in HDAS (defined as DAS28ESR > 5.1) [7], and intensive physical therapy [8].
To this end, we undertook a retrospective analysis using records of patients with RA in persistent MDAS managed using a T2T approach in a routine UK national health service setting. Our primary aim was to identify factors predicting patient functional outcomes at 12 months. Secondary aims were to identify factors predicting patient HRQoL at 12 months and to characterise pain, disability, and HRQoL in patients in persistent MDAS.

\section{Methods \\ Subjects}

We retrospectively evaluated patient records from Guy's Hospital RA Centre, which maintains an electronic healthcare record cohort of patients attending routine rheumatology appointments at Guy's Hospital (South London) $[9,10]$. Since inception of the database in 2006, clinician- and patient-reported outcomes, including DAS28-ESR, Health Assessment QuestionnaireDisability Index (HAQ-DI) [11] and EuroQol 5dimensions 3-level questionnaire index (EQ-5D-3L) scores (UK value set) [12], have been routinely recorded for patients at each clinic visit. All patients are managed in line with T2T recommendations.

No globally accepted definition of persistent MDAS exists, with previous research in this area using varying descriptions $[4,6]$. In this analysis, we considered patients to be in persistent MDAS if they had DAS28-ESR scores of 3.2-5.1 on at least two consecutive outpatient appointments over a 12-month period. For the purposes of this study, patients also had to have recorded HAQDI scores at both baseline and final timepoints (to allow the primary study aim to be addressed).

Patients in persistent MDAS were analysed in two separate groups: first, those naïve to biologic DMARDs at baseline and, second, those who were receiving or had previously received biologic DMARDs. The rationale for this sub-categorisation of patients was that the next step 
in the therapeutic pathway could differ between these patient groups.

\section{Statistical analysis}

For this analysis, the baseline timepoint was the visit at which patients met our definition of 'persistent MDAS'. Thus, the baseline timepoint was the second of two consecutive outpatient appointments over a 12-month period in which each patient had a DAS28-ESR score of $3.2-5.1$. The endpoint was the outpatient visit closest to 12 months after the baseline visit.

Linear regression analyses were used to explore relationships between baseline variables and (1) 12-month HAQ-DI scores, (2) 12-month EQ-5D-3L index scores, (3) 12-month changes in HAQ-DI scores, and (4) 12month changes in EQ-5D-3L index scores. Ranktransformed 12-month EQ-5D-3L index scores were used, as raw 12-month EQ-5D-3L index scores were not normally distributed. This involved the use of an ordered quantile normalisation transformation approach, a rankbased procedure whereby the values of a vector are mapped to their percentile, which is then mapped to the same percentile of the normal distribution [13].

Baseline variables evaluated in the linear regression models comprised age, sex, ethnicity, disease duration, rheumatoid factor (RF) status, RA therapy (monotherapy or combination synthetic DMARD therapy or no treatment for biologic-naïve patients; DMARD monotherapy, biologic monotherapy, or combination synthetic DMARD and biologic therapy for biologic-experienced patients), corticosteroid use, ESR, DAS28-ESR, swollen joint count (SJC), tender joint count (TJC), HAQ-DI score, Patient Global Assessment of Disease Activity (PtGA), EQ-5D-3L index score, EQ-5D-3L pain score (question 4 of the EQ-5D-3L), and EQ-5D-3L anxiety/depression score (question 5 of the EQ-5D-3L). Due to substantial levels of missing data, we did not include anti-cyclic citrullinated peptide or C-reactive protein levels in the linear regression models, opting for RF and ESR to capture information on serology and the acute-phase response instead.

Univariate linear regression models included either (1) 12-month HAQ-DI scores, (2) 12-month ranktransformed EQ-5D-3L index scores, (3) 12-month change in HAQ-DI scores, or (4) 12-month change in EQ-5D-3L index scores as the response variable and each individual baseline variable as the explanatory variable. Baseline variables with a $P$-value of $<0.1$ in univariate models were subsequently included in multivariate regression models as explanatory variables.

Correlation coefficients for variables that may have been affected by strong multicollinearity (DAS28 and its components; EQ-5D-3L index scores and EQ-5D-3L pain and depression/anxiety scores) were checked to ensure they did not meet the pre-defined threshold of $>0.7$, which would have precluded entry of both variables into the same model.

For all analyses, $P$-values of $<0.05$ were considered statistically significant; no multiplicity adjustments were undertaken. Missing data were not imputed. All analyses were performed using $\mathrm{R}$ (version 3.5.3). Rank-based transformations were undertaken using the $\mathrm{R}$ package 'bestNormalise' [13].

\section{Results}

\section{Baseline characteristics}

From 17,002 patient-visits, we identified 422 patients with RA who met our criteria for persistent MDAS. Of these, 395 had HAQ-DI scores at baseline and final timepoints, and were included in the analysis (207 biologic-naïve and 188 biologic-experienced patients).

In both patient groups, baseline HAQ-DI and EQ-5D-3L index scores indicated moderate disability and reduced HRQoL (Table 1). The majority of patients reported moderate or severe pain on the EQ-5D-3L pain scale at baseline (93\% of biologic-naïve and 96\% of biologic-experienced patients). Compared with biologic-naïve patients, biologicexperienced patients were younger, showed longer RA duration, were more likely to be RF positive, and had higher rates of corticosteroid use. They also had higher DAS28ESR and HAQ-DI scores and were more likely to report moderate or severe anxiety/depression at study baseline.

\section{Changes in HAQ-DI scores}

Baseline and 12-month mean HAQ-DI scores were similar in the two patient groups. In biologic-naïve patients, baseline and 12-month mean HAQ-DI scores were $1.21 \pm 0.79$ (standard deviation [SD]) and $1.21 \pm 0.80$, respectively. Many patients showed substantial changes in HAQ-DI scores over the follow-up period (Supplementary Fig. 1). A worsening or improvement in HAQ-DI scores that exceeded the minimum clinically important difference (MCID) of 0.22 units [14] was observed in 60 (29\%) and 58 (28\%) patients, respectively. In biologic-experienced patients, baseline and 12month mean HAQ-DI scores were $1.46 \pm 0.74$ and $1.47 \pm$ 0.76 , respectively. A worsening or improvement in HAQ-DI scores that exceeded the MCID of 0.22 units was observed in $56(30 \%)$ and $50(27 \%)$ patients, respectively.

\section{Changes in EQ-5D-3L index scores}

As with HAQ-DI scores, baseline and 12-month mean EQ-5D-3L index scores were similar in both patient groups. In biologic-naïve patients, baseline and 12month mean EQ-5D-3L index scores were $0.52 \pm 0.30$ (SD) and $0.53 \pm 0.30$, respectively; mean change in EQ5D-3L index scores was $0.02 \pm 0.31$, reflecting an improvement in QoL. In biologic-experienced patients, baseline and 12-month mean EQ-5D-3L index scores were 
Table 1 Baseline characteristics of patients with RA with persistent moderate disease activity

\begin{tabular}{|c|c|c|c|}
\hline Baseline variable & & $\begin{array}{l}\text { Biologic naïve } \\
(N=207)\end{array}$ & $\begin{array}{l}\text { Biologic experienced } \\
(N=188)\end{array}$ \\
\hline Age (years) & & $58.5 \pm 15.6$ & $55.4 \pm 14.1$ \\
\hline Female & & $167(81)$ & $153(81)$ \\
\hline \multirow[t]{5}{*}{ Ethnicity } & White & $140(70)$ & $112(74)$ \\
\hline & Black & $33(17)$ & $25(17)$ \\
\hline & Asian & $12(6)$ & $6(4)$ \\
\hline & Mixed & $4(2)$ & $3(2)$ \\
\hline & Other & $9(5)$ & $5(3)$ \\
\hline Duration of RA (years) & & $7.0 \pm 9.0$ & $21.1 \pm 12.0$ \\
\hline RF positive & & $105(64)$ & $131(80)$ \\
\hline Anti-CCP positive & & $69(54)$ & $65(59)$ \\
\hline Corticosteroids & & $22(11)$ & $38(20)$ \\
\hline \multirow[t]{3}{*}{ Treatment: Biologic naïve } & Monotherapy & $99(52)$ & - \\
\hline & Combination & $89(47)$ & - \\
\hline & None & $3(2)$ & - \\
\hline \multirow[t]{3}{*}{ Treatment: Biologic experienced } & DMARD & - & $51(30)$ \\
\hline & Biologic & - & $25(15)$ \\
\hline & DMARD - biologic & - & $94(55)$ \\
\hline DAS28-ESR & & $4.0 \pm 0.5$ & $4.1 \pm 0.5$ \\
\hline 28-TJC & & $4.3 \pm 4.3$ & $4.9 \pm 4.2$ \\
\hline $28-S J C$ & & $2.1 \pm 2.8$ & $2.7 \pm 2.6$ \\
\hline PtGA & & $50.0 \pm 21.8$ & $51.0 \pm 21.4$ \\
\hline ESR & & $21.8 \pm 16.3$ & $20.0 \pm 16.1$ \\
\hline HAQ-DI & & $1.2 \pm 0.8$ & $1.5 \pm 0.7$ \\
\hline EQ-5D-3L index & & $0.5 \pm 0.3$ & $0.5 \pm 0.3$ \\
\hline \multirow[t]{3}{*}{ EQ-5D-3L pain } & None & $12(7)$ & $8(4)$ \\
\hline & Moderate & $135(79)$ & $153(82)$ \\
\hline & Severe & $23(14)$ & $26(14)$ \\
\hline \multirow[t]{3}{*}{ EQ-5D-3L anxiety/depression } & None & $92(55)$ & $91(49)$ \\
\hline & Moderate & $64(38)$ & $87(47)$ \\
\hline & Severe & $12(7)$ & $8(4)$ \\
\hline
\end{tabular}

Results are presented as $\mathrm{n}(\%)$ or mean \pm standard deviation. The following data are missing in the biologic-naïve population: ethnicity in 9 patients; disease duration in 7 patients; RF in 43 patients; anti-CCP in 80 patients; DMARD treatment in 16 patients; TJC/SJC/PtGA in 1 patient; ESR in 6 patients; EQ-5D-3L index scores in 35 patients. The following data are missing in the biologic-experienced population: sex in 2 patients; ethnicity in 37 patients; RF in 25 patients; anti-CCP in 78 patients; DMARD treatment in 18 patients; PtGA in 1 patient; ESR in 12 patients; EQ-5D-3L index scores in 1 patient

Abbreviations: CCP cyclic citrullinated peptide antibody, DAS28-ESR Disease Activity Score for 28-joint count based on erythrocyte sedimentation rate, DMARD disease-modifying anti-rheumatic drug, EQ-5D-3L 3-level EuroQol 5-dimensions questionnaire, ESR erythrocyte sedimentation rate, $H A Q-D I$ Health Assessment Questionnaire-Disability Index, PtGA patient global assessment, RA rheumatoid arthritis, RF rheumatoid factor, SJC swollen joint count, TJC tender joint count

$0.50 \pm 0.26$ and $0.49 \pm 0.31$, respectively; mean change in EQ$5 \mathrm{D}-3 \mathrm{~L}$ index scores was minimal $(-0.005 \pm 0.31)$. Despite small changes in mean scores, histograms demonstrated that many patients showed substantial changes in EQ-5D-3L index scores over the follow-up period (Supplementary Fig. 1).

\section{Predictors of endpoint HAQ-DI scores}

In univariate analysis in the biologic-naïve group, baseline age, male sex, disease duration, RF, DAS28-ESR, SJC, PtGA, HAQ-DI, EQ-5D-3L index scores, EQ-5D-3L pain scores, and EQ-5D-3L anxiety/depression scores all showed significant associations $(P<0.05)$ with 12 -month HAQ-DI scores (Table 2). In the biologic-experienced group, age, corticosteroid use, SJC, PtGA, HAQ-DI, EQ5D-3L index scores, EQ-5D-3L pain scores, and EQ-5D$3 \mathrm{~L}$ anxiety/depression scores all showed significant associations $(P<0.05)$ with 12 -month HAQ-DI scores (Table 3).

In multivariate analysis in both patient groups, only baseline HAQ-DI score showed a significant association with 12- 
Table 2 Association of baseline variables with HAQ-DI and EQ-5D-3L index scores in biologic DMARD-naïve patients

\begin{tabular}{|c|c|c|c|c|c|c|c|c|c|c|c|c|c|c|c|c|}
\hline \multirow[t]{3}{*}{$\begin{array}{l}\text { Baseline } \\
\text { variable }\end{array}$} & \multicolumn{4}{|c|}{ 12-month HAQ-DI score } & \multicolumn{4}{|c|}{$\begin{array}{l}\text { 12-month change in HAQ-DI } \\
\text { score }\end{array}$} & \multicolumn{4}{|c|}{ 12-month EQ-5D-3L score } & \multicolumn{4}{|c|}{$\begin{array}{l}\text { 12-month change in EQ-5D-3L } \\
\text { score }\end{array}$} \\
\hline & \multicolumn{2}{|c|}{ Univariate } & \multicolumn{2}{|c|}{ Multivariate } & \multicolumn{2}{|c|}{ Univariate } & \multicolumn{2}{|c|}{ Multivariate } & \multicolumn{2}{|c|}{ Univariate } & \multicolumn{2}{|c|}{ Multivariate } & \multicolumn{2}{|c|}{ Univariate } & \multicolumn{2}{|c|}{ Multivariate } \\
\hline & $\bar{\beta}$ & $P$ & $\bar{\beta}$ & $P$ & $\bar{\beta}$ & $P$ & $\bar{\beta}$ & $P$ & $\beta$ & $P$ & $\beta$ & $P$ & $\beta$ & $P$ & $\beta$ & $P$ \\
\hline Age & 0.01 & 0.017 & 0.00 & 0.284 & 0.00 & 0.084 & 0.00 & 0.035 & 0.00 & 0.595 & - & - & 0.00 & 0.849 & - & - \\
\hline Male sex & -0.36 & 0.010 & -0.19 & 0.134 & -0.01 & 0.948 & - & - & 0.24 & 0.182 & - & - & 0.30 & 0.149 & - & - \\
\hline White & Ref & - & - & - & Ref & - & - & - & Ref & - & Ref & - & Ref & - & Ref & - \\
\hline Black & 0.25 & 0.105 & - & - & -0.06 & 0.547 & - & - & -0.52 & 0.013 & -0.44 & 0.017 & -0.12 & 0.086 & -0.16 & 0.010 \\
\hline Asian & -0.39 & 0.113 & - & - & -0.15 & 0.354 & - & - & 0.34 & 0.246 & -0.15 & 0.571 & 0.07 & 0.495 & 0.06 & 0.506 \\
\hline Mixed & -0.17 & 0.675 & - & - & -0.04 & 0.888 & - & - & 0.47 & 0.329 & 0.21 & 0.610 & 0.24 & 0.139 & 0.16 & 0.268 \\
\hline Other & 0.16 & 0.571 & - & - & 0.17 & 0.357 & - & - & -0.61 & 0.100 & -0.52 & 0.095 & -0.08 & 0.523 & -0.13 & 0.207 \\
\hline $\begin{array}{l}\text { Disease } \\
\text { duration }\end{array}$ & 0.01 & 0.037 & 0.01 & 0.208 & 0.01 & 0.197 & - & - & 0.00 & 0.941 & - & - & 0.00 & 0.854 & - & - \\
\hline RF positive & -0.45 & $<0.001$ & -0.19 & 0.051 & -0.06 & 0.486 & - & - & 0.07 & 0.708 & - & - & -0.10 & 0.102 & - & - \\
\hline Corticosteroids & -0.02 & 0.906 & - & - & 0.05 & 0.685 & - & - & 0.23 & 0.365 & - & - & 0.30 & 0.710 & - & - \\
\hline DAS28-ESR & 0.21 & 0.048 & -0.01 & 0.881 & -0.08 & 0.254 & - & - & -0.05 & 0.708 & - & - & 0.05 & 0.282 & - & - \\
\hline $\begin{array}{l}\text { Swollen joint } \\
\text { count }\end{array}$ & -0.06 & 0.002 & -0.02 & 0.227 & -0.01 & 0.415 & - & - & 0.08 & 0.006 & 0.04 & 0.184 & 0.00 & 0.664 & - & - \\
\hline $\begin{array}{l}\text { Tender joint } \\
\text { count }\end{array}$ & 0.01 & 0.637 & - & - & 0.00 & 0.926 & - & - & 0.00 & 0.853 & - & - & 0.00 & 0.576 & - & - \\
\hline PtGA & 0.01 & $<0.001$ & 0.00 & 0.225 & 0.00 & 0.008 & 0.00 & 0.643 & -0.01 & $<0.001$ & 0.00 & 0.502 & 0.00 & 0.069 & 0.00 & 0.197 \\
\hline ESR & 0.00 & 0.626 & - & - & 0.00 & 0.971 & - & - & 0.00 & 0.678 & - & - & 0.00 & 0.662 & - & - \\
\hline $\begin{array}{l}\text { DMARD } \\
\text { monotherapy }\end{array}$ & Ref & - & - & - & Ref & - & - & - & Ref & - & Ref & - & Ref & - & - & - \\
\hline $\begin{array}{l}\text { DMARD } \\
\text { combination }\end{array}$ & 0.05 & 0.646 & - & - & 0.07 & 0.340 & - & - & -0.40 & 0.008 & -0.44 & 0.002 & -0.08 & 0.138 & - & - \\
\hline No DMARDs & -0.65 & 0.171 & - & - & -0.10 & 0.738 & - & - & -0.01 & 0.989 & -0.48 & 0.300 & -0.26 & 0.160 & - & - \\
\hline HAQ-DI & 0.79 & $<0.001$ & 0.67 & $<0.001$ & -0.21 & $<0.001$ & -0.21 & $<0.001$ & -0.65 & $<0.001$ & -0.57 & $<0.001$ & 0.01 & 0.739 & - & - \\
\hline $\begin{array}{l}\text { EQ-5D-3L } \\
\text { index }\end{array}$ & -1.30 & $<0.001$ & -0.45 & 0.119 & 0.00 & 0.977 & - & - & 1.52 & $<0.001$ & 0.27 & 0.527 & -0.54 & $<0.001$ & -0.73 & $<0.001$ \\
\hline $\begin{array}{l}\text { EQ-5D-3L } \\
\text { pain }\end{array}$ & 0.47 & $<0.001$ & -0.17 & 0.272 & -0.09 & 0.347 & - & - & -0.76 & $<0.001$ & -0.11 & 0.623 & 0.20 & $<0.001$ & -0.05 & 0.497 \\
\hline $\begin{array}{l}\text { EQ-5D-3L } \\
\text { anxiety/ } \\
\text { depression }\end{array}$ & 0.34 & $<0.001$ & 0.11 & 0.242 & 0.003 & 0.621 & - & - & -0.56 & $<0.001$ & -0.56 & 0.089 & 0.10 & 0.008 & -0.04 & 0.353 \\
\hline
\end{tabular}

$\beta$ - and $P$-values are from linear regression models that included endpoint HAQ-DI score, endpoint EQ-5D-3L index score (rank-transformed), 12-month change in HAQ-DI score or 12-month change in EQ-5D-3L index score as the response variable and each baseline variable as the explanatory variable (univariate model) or all baseline variables with $P$-values $<0.1$ from univariate analysis (multivariate model); $P$-values shown in bold are significant $(<0.05)$

Abbreviations: DAS28-ESR Disease Activity Score for 28-joint count based on erythrocyte sedimentation rate, DMARD disease-modifying anti-rheumatic drug, EQ-5D$3 L$ 3-level EuroQol 5-Dimensions questionnaire, ESR erythrocyte sedimentation rate, HAQ-DI Health Assessment Questionnaire-Disability Index, PtGA Patient Global Assessment of Disease Activity on a $10 \mathrm{~cm}$ visual analogue scale, Ref reference group, $R F$ rheumatoid factor

month HAQ-DI scores, with higher baseline HAQ-DI scores being associated with higher 12-month HAQ-DI scores, indicating greater disability (Tables 2 and 3, and Fig. 1). In biologic-naïve patients, the $\beta$-value of 0.67 (standard error [SE] 0.07) indicated that the 12-month HAQ-DI score was 0.67 units higher per unit increase in baseline HAQ-DI score. In biologic-experienced patients, the $\beta$-value of 0.76 (SE 0.06 ) indicated that the 12-month HAQ-DI score was 0.76 units higher per unit increase in baseline HAQ-DI score.

\section{Predictors of change in HAQ-DI scores}

In univariate analysis in the biologic-naïve group, age, PtGA, and HAQ-DI scores all showed significant associations $(P<0.05)$ with 12 -month changes in HAQ-DI scores (Table 2), whereas variables associated with such changes in the biologic-experienced group were DAS28ESR, TJC, PtGA, and HAQ-DI scores (Table 3).

In multivariate analysis in both patient groups, baseline HAQ-DI score showed a significant association with 
Table 3 Association of baseline variables with HAQ-DI and EQ-5D-3L index scores in biologic DMARD-experienced patients

\begin{tabular}{|c|c|c|c|c|c|c|c|c|c|c|c|c|c|c|c|c|}
\hline \multirow[t]{3}{*}{ Baseline variable } & \multicolumn{4}{|c|}{ 12-month HAQ-DI score } & \multicolumn{4}{|c|}{$\begin{array}{l}\text { 12-month change in HAQ-DI } \\
\text { score }\end{array}$} & \multicolumn{4}{|c|}{ 12-month EQ-5D-3L score } & \multicolumn{4}{|c|}{$\begin{array}{l}\text { 12-month change in EQ-5D-3L } \\
\text { score }\end{array}$} \\
\hline & \multicolumn{2}{|c|}{ Univariate } & \multicolumn{2}{|c|}{ Multivariate } & \multicolumn{2}{|c|}{ Univariate } & \multicolumn{2}{|c|}{ Multivariate } & \multicolumn{2}{|c|}{ Univariate } & \multicolumn{2}{|c|}{ Multivariate } & \multicolumn{2}{|c|}{ Univariate } & \multicolumn{2}{|c|}{ Multivariate } \\
\hline & $\bar{\beta}$ & $P$ & $\bar{\beta}$ & $P$ & $\bar{\beta}$ & $P$ & $\bar{\beta}$ & $P$ & $\bar{\beta}$ & $P$ & $\bar{\beta}$ & $P$ & $\bar{\beta}$ & $P$ & $\bar{\beta}$ & $P$ \\
\hline Age & 0.01 & 0.003 & 0.00 & 0.554 & 0.00 & 0.698 & - & - & 0.00 & 0.510 & - & - & 0.00 & 0.510 & - & - \\
\hline Male sex & -0.06 & 0.679 & - & - & 0.07 & 0.468 & - & - & -0.30 & 0.119 & - & - & 0.01 & 0.886 & - & - \\
\hline White & Ref & - & - & - & Ref & - & - & - & Ref & - & Ref & - & Ref & - & Ref & - \\
\hline Black & -0.23 & 0.173 & - & - & -0.08 & 0.527 & - & - & 0.12 & 0.574 & - & - & 0.03 & 0.620 & - & - \\
\hline Asian & -0.39 & 0.218 & - & - & -0.10 & 0.658 & - & - & 0.17 & 0.672 & - & - & -0.08 & 0.542 & - & - \\
\hline Mixed & -0.43 & 0.328 & - & - & -0.07 & 0.813 & - & - & 0.66 & 0.341 & - & - & 0.05 & 0.815 & - & - \\
\hline Other & -0.05 & 0.888 & - & - & -0.26 & 0.291 & - & - & 0.51 & 0.255 & - & - & 0.23 & 0.106 & - & - \\
\hline Disease duration & 0.00 & 0.667 & - & - & 0.00 & 0.845 & - & - & 0.01 & 0.395 & - & - & 0.00 & 0.862 & - & - \\
\hline RF positive & -0.14 & 0.342 & - & - & -0.12 & 0.249 & - & - & 0.12 & 0.535 & - & - & 0.02 & 0.730 & - & - \\
\hline Corticosteroids & 0.31 & 0.026 & 0.09 & 0.369 & 0.00 & 0.968 & - & - & -0.12 & 0.490 & - & - & 0.03 & 0.572 & - & - \\
\hline DAS28-ESR & 0.00 & 0.986 & - & - & -0.14 & 0.057 & 0.01 & 0.891 & 0.00 & 0.990 & - & - & 0.09 & 0.053 & 0.05 & 0.269 \\
\hline $\begin{array}{l}\text { Swollen joint } \\
\text { count }\end{array}$ & -0.04 & 0.049 & -0.01 & 0.391 & 0.00 & 0.991 & - & - & 0.04 & 0.138 & - & - & 0.01 & 0.510 & - & - \\
\hline $\begin{array}{l}\text { Tender joint } \\
\text { count }\end{array}$ & -0.02 & 0.139 & - & - & -0.02 & 0.001 & -0.03 & 0.006 & 0.01 & 0.572 & - & - & 0.01 & 0.161 & - & - \\
\hline PtGA & 0.01 & 0.014 & 0.00 & 0.401 & 0.00 & 0.060 & 0.00 & 0.421 & -0.01 & 0.028 & 0.00 & 0.850 & 0.00 & 0.047 & 0.00 & 0.293 \\
\hline ESR & 0.00 & 0.628 & - & - & 0.00 & 0.754 & - & - & 0.00 & 0.222 & - & - & 0.00 & 0.774 & - & - \\
\hline DMARD & Ref & - & - & - & Ref & - & - & - & Ref & - & Ref & - & Ref & - & Ref & - \\
\hline Biologic & 0.11 & 0.561 & - & - & 0.08 & 0.530 & - & - & 0.08 & 0.899 & - & - & -0.04 & 0.604 & - & - \\
\hline DMARD biologic & 0.02 & 0.902 & - & - & -0.01 & 0.882 & - & - & -0.02 & 0.899 & - & - & -0.04 & 0.472 & - & - \\
\hline HAQ-DI & 0.79 & $<0.001$ & 0.76 & $<0.001$ & -0.21 & $<0.001$ & -0.21 & $<0.001$ & -0.49 & $<0.001$ & -0.29 & 0.004 & 0.03 & 0.283 & - & - \\
\hline EQ-5D-3L index & -1.08 & $<0.001$ & -0.02 & 0.949 & 0.28 & 0.053 & -0.10 & 0.546 & 1.61 & $<0.001$ & 1.18 & 0.007 & -0.50 & $<0.001$ & -0.40 & 0.003 \\
\hline EQ-5D-3L pain & 0.33 & 0.012 & 0.01 & 0.935 & -0.11 & 0.211 & - & - & -0.53 & 0.002 & 0.23 & 0.325 & 0.29 & $<0.001$ & 0.13 & 0.090 \\
\hline $\begin{array}{l}\text { EQ-5D-3L } \\
\text { anxiety/ } \\
\text { depression }\end{array}$ & 0.23 & 0.019 & 0.05 & 0.544 & -0.01 & 0.875 & - & - & -0.54 & $<0.001$ & -0.25 & 0.058 & 0.09 & 0.026 & -0.02 & 0.674 \\
\hline
\end{tabular}

$\beta$ - and $P$-values are from linear regression models that included endpoint HAQ-DI score, endpoint EQ-5D-3L index score (rank-transformed), 12 -month change in HAQ-DI score or 12-month change in EQ-5D-3L index score as the response variable and each baseline variable as the explanatory variable (univariate model) or all baseline variables with $P$-values $<0.1$ from univariate analysis (multivariate model); $P$-values shown in bold are significant $(<0.05$ )

Abbreviations: DAS28-ESR Disease Activity Score for 28-joint count based on erythrocyte sedimentation rate, DMARD disease-modifying anti-rheumatic drug, EQ-5D$3 L$ 3-level EuroQol 5-Dimensions questionnaire, ESR erythrocyte sedimentation rate, HAQ-DI Health Assessment Questionnaire-Disability Index, PtGA Patient Global Assessment of Disease Activity on a $10 \mathrm{~cm}$ visual analogue scale, Ref reference group, RF Rheumatoid factor

12-month changes in HAQ-DI scores, with higher baseline HAQ-DI scores predicting greater 12-month reductions (improved functioning) in HAQ-DI scores (Tables 2 and 3, and Fig. 1). In both biologic-naïve and biologicexperienced patients, the $\beta$-values of -0.21 (SE 0.05) and -0.21 (SE 0.06), respectively, indicated that the 12month reduction in HAQ-DI score was 0.21 units greater per unit increase in the baseline HAQ-DI score. Significant associations with 12-month changes in HAQDI scores were also observed for age $(P=0.035)$ in biologic-naïve patients and for TJC $(P=0.006)$ in biologic-experienced patients; the small $\beta$-values $(0.005$ and -0.03 , respectively) indicated uncertain clinical relevance for these statistical associations.

\section{Predictors of endpoint EQ-5D-3L index scores}

In univariate analysis in both patient groups, PtGA, HAQDI, EQ-5D-3L index scores, EQ-5D-3L pain scores, and EQ-5D-3L anxiety/depression scores all showed significant associations $(P<0.05)$ with 12 -month EQ-5D-3L index scores (Tables 2 and 3; Fig. 2). In the biologic-naïve group, Black ethnicity, the use of combination DMARD therapy, and SJC also showed significant associations.

In multivariate analysis, baseline HAQ-DI scores were significantly associated with 12-month EQ-5D-3L index scores in both biologic-naive $(\beta=-0.57$; SE 0.10$)$ and biologic-experienced patients $(\beta=-0.29)$. It was not possible to clinically interpret the $\beta$-values from the regression models because of the rank-transformation of 


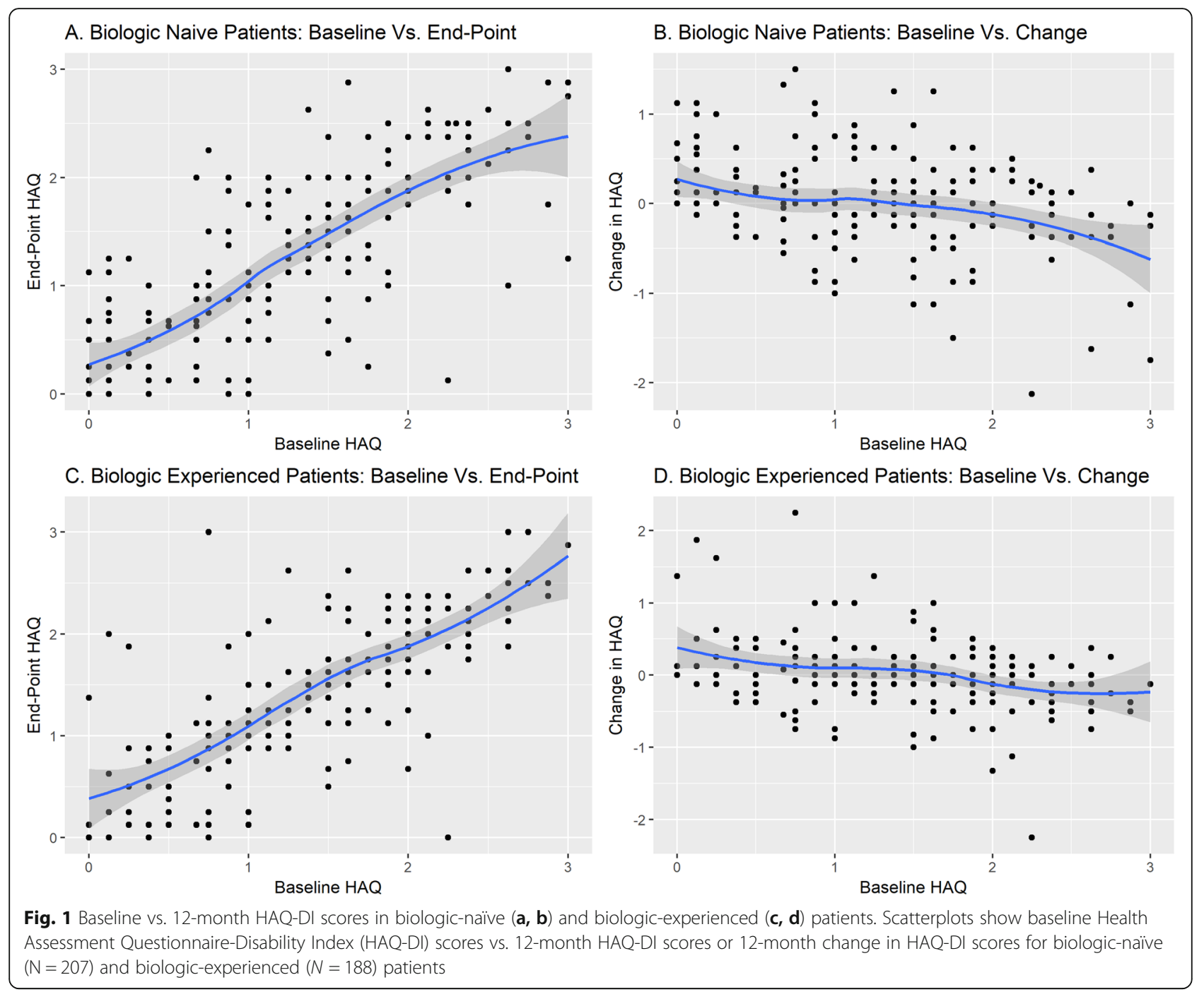

12-month EQ-5D-3L index scores. Twelve-month EQ5D-3L index scores had significant associations with Black ethnicity and combination DMARD therapy in biologic-naïve patients and with baseline EQ-5D-3L index scores in biologic-experienced patients.

\section{Predictors of change in EQ-5D-3L index scores}

In univariate analysis in both patient groups, EQ-5D-3L index scores, EQ-5D-3L pain scores, and EQ-5D-3L anxiety/depression scores all showed significant associations $(P<0.05)$ with 12 -month changes in EQ-5D-3L index scores (Tables 2 and 3; Fig. 2). PtGA also showed a significant association in the biologic-experienced group.

In multivariate analysis, baseline EQ-5D-3L scores were significantly associated with 12-month changes in EQ-5D$3 \mathrm{~L}$ index scores in both patient groups. In biologic-naïve patients, the $\beta$-value of -0.73 (SE 0.14) indicated that the 12 -month decrease in EQ-5D-3L index scores was 0.73 units more per unit increase in the baseline EQ-5D-3L index score. In biologic-experienced patients, the $\beta$-value of -0.40 (SE 0.13) indicated that the 12-month decrease in EQ-5D-3L index scores was 0.40 units more per unit increase in the baseline EQ-5D-3L index score. In biologicnaïve patients, Black ethnicity also showed a significant association with 12-month changes in EQ-5D-3L index scores $(\beta=-0.16 ; P=0.010)$.

\section{Discussion}

Our retrospective analysis of patients with persistent MDAS in a real-world, T2T setting has three key findings. First, it shows HAQ-DI is a key predictor of patients' future functional status and HRQoL, with baseline HAQ-DI scores significantly associated with 12month HAQ-DI and EQ-5D-3L index scores in both biologic-naïve and biologic-experienced patients. Second, it supports existing research indicating that MDAS is not a benign disease activity state; many patients evaluated in this study had substantial ongoing impaired 


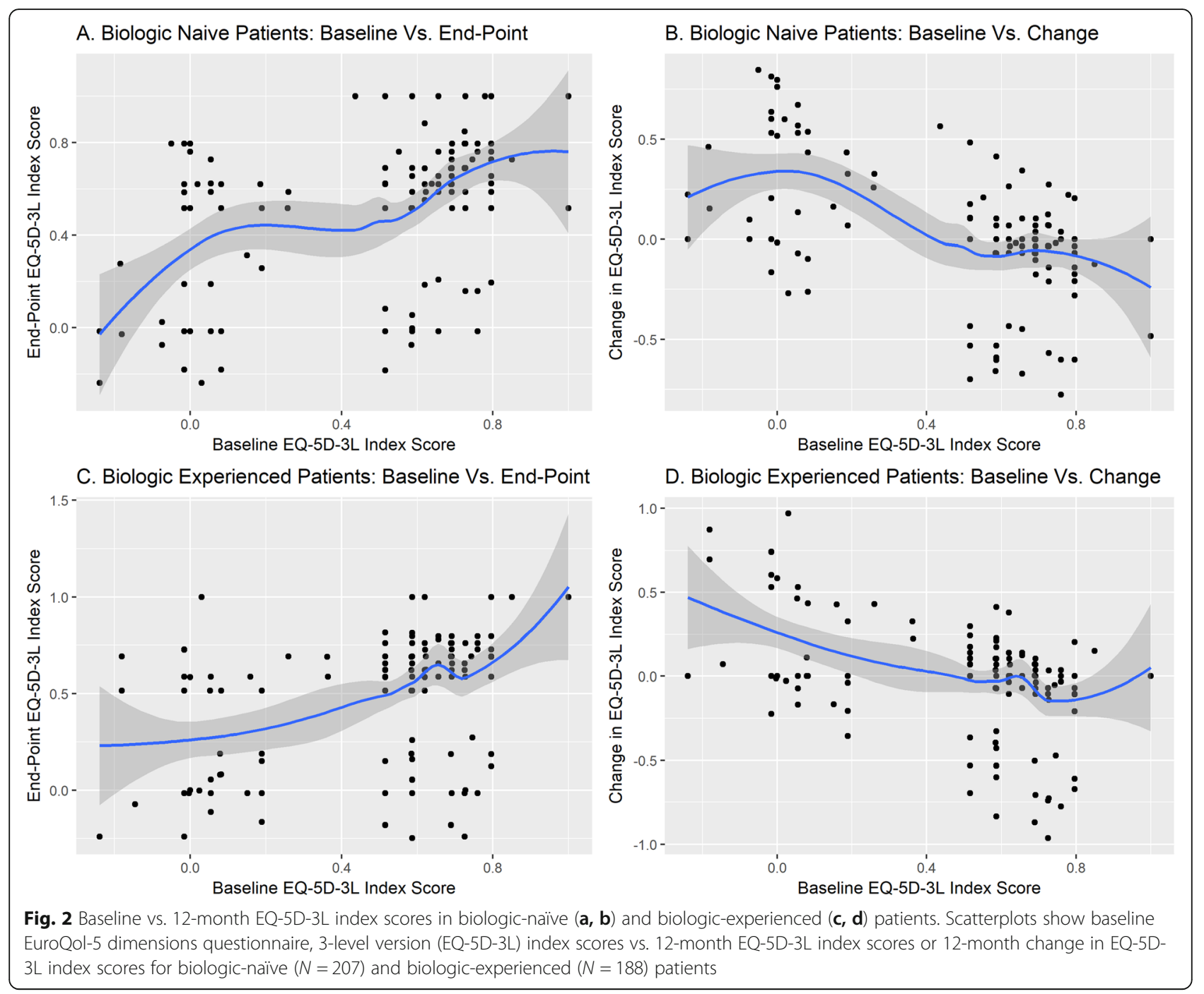

physical function and HRQoL. Third, it highlights the ongoing unmet need to address pain in patients with RA; over $90 \%$ of patients reported moderate or severe pain when assessed using the EQ-5D-3L. Overall, these findings support the T2T strategy of aiming for remission/LDAS [2,3] and the current UK National Institute for Health and Care Excellence (NICE) guidelines advocating use of the HAQ-DI in routine care [15]. Achieving MDAS alone appears insufficient to optimise pain relief, physical function, and HRQoL.

Over 12 months, mean HAQ-DI scores were both relatively high and relatively static in patients with MDAS. This finding is consistent with data from the Yorkshire Early Arthritis Register, which showed that persistent MDAS was associated with persistently high HAQ-DI scores in early RA [6]. However, a minority of individuals did demonstrate large changes in their HAQ-DI scores, reflecting evidence that individual patients have different HAQ-DI trajectories, both in early disease [16] and in persistent MDAS after biologic therapy [17]. An associated finding in our study was that initial HAQ-DI scores strongly predicted final HAQ-DI scores, patients with worse baseline function tending to have worse function at study endpoint. This finding reflects the considerable evidence that baseline HAQ-DI scores are important predictors of subsequent outcomes, including future disability. Research on HAQ-DI as a predictor of outcomes has been mainly performed in early RA cohorts [18-21], though there is evidence that baseline HAQ-DI scores are also predictors in established RA [22]. Our further finding that higher baseline HAQ-DI scores were significantly associated with greater 12month reductions in HAQ-DI scores, which appears counterintuitive, is most likely explained by 'regression to the mean'. This well-established and ubiquitous statistical phenomenon occurs when unusually large or small 
measurements tend to be followed by measurements that are closer to the mean [23], and is seen throughout studies using repeated measures, including birthweight of subsequent childbirths [24], blood pressure [25], bone mineral density scores [26] and cholesterol levels [27].

We found that most MDAS patients (93 and 96\% of biologic DMARD-naïve and biologic DMARD-experienced patients, respectively) had moderate to severe pain on the EQ-5D-3L pain scale at baseline. This supports existing patient survey data highlighting that pain is a significant unmet need for patients with RA, with many patients reporting pain to be their preferred area for improvement in the management of their disease [28-30]. Pain in people with RA is a multidimensional experience driven by a broad range of factors, including disease activity, pain pathway sensitisation, joint damage, and the health beliefs of patients [31]. The cornerstone of pain management lies in its assessment, with Chua et al. [32] recommending the collection of pain scores at all clinic assessments. Our data support their suggestion, and further underline the importance of improving how pain is managed in people with RA.

Our study has several strengths. These include the reallife T2T clinical setting, the focus on a previously poorly studied patient population in persistent MDAS, and the comprehensive prospective capture of clinical data over an extended period. It also has several limitations. First, the use of data from a single centre resulted in a relatively limited sample size. In addition, these data may not be representative of all rheumatology centres in the UK. Small sample sizes are an inevitable consequence of single-centre studies, since the numbers of patients in specific disease activity groups are likely to be restricted. However, single centres can also achieve a consistent management approach, which may be diluted in multicentre observational studies. Second, as these data were captured in a real-life setting when patients routinely attended the rheumatology clinic rather than scheduled study visits, there was also some variability in the times at which outcome variables were measured. Such variability is unavoidable in real-life settings. Third, pain was assessed using the EQ-5D-3L pain scale, which only evaluates one facet of a multidimensional problem using a limited number of pain categories.

\section{Conclusion}

The HAQ-DI is an important predictor of future function and HRQoL. Our study underlines the need to supplement disease activity assessments with HAQ-DI measurements (in-line with existing NICE guidelines), to provide important insight into which patients are likely to demonstrate poor future function and reduced HRQoL. It also provides further evidence that persistent MDAS is not a 'benign' disease activity state, with many patients in our study experiencing substantial physical disability and poor HRQoL alongside high pain levels.

\section{Supplementary information}

Supplementary information accompanies this paper at https://doi.org/10. 1186/s41927-020-00161-4.

Additional file 1 Supplementary Fig. 1. Changes in study variables in biologic-naïve (A, B, C) and biologic-experienced (C, D, E) patients. Legend: Histograms show 12-month changes in DAS28-ESR, HAQ-DI, and EQ-5D-3L index scores in biologic-naïve $(N=207)$ and biologicexperienced $(N=188)$ patients.

\section{Abbreviations}

DAS28: Disease Activity Score for 28-joint count; DAS28-ESR: Disease Activity Score for 28-joint count based on erythrocyte sedimentation rate; DMARD: Disease-modifying anti-rheumatic drug; EQ-5D-3L: 3-level version of the EuroQol 5-dimensions questionnaire; ESR: Erythrocyte sedimentation rate; HAQ-DI: Health Assessment Questionnaire-Disability Index; HDAS: High disease activity state; HRQoL: Health-related quality of life; LDAS: Low disease activity state; MCID: Minimum clinically important difference; MDAS: Moderate disease activity state; NICE: National Institute for Health and Care Excellence; PtGA: Patient Global Assessment of Disease Activity; QoL: Quality of life; RA: Rheumatoid arthritis; RF: Rheumatoid factor; RAPID3: Routine Assessment of Patient Index Data 3; SD: Standard deviation; SE: Standard error; SJC: Swollen joint count; TJC: Tender joint count; T2T: Treat-to-target

\section{Acknowledgements}

Medical writing services were provided by Dr. Sue Chambers and Karen Goa (Rx Communications, Mold, UK) and were funded by Eli Lilly and Company.

\section{Authors' contributions}

$J M, J B$ and BK conceived the study idea; ICS undertook the statistical analysis and drafted the manuscript; all authors revised the manuscript and approved the final submitted version.

\section{Funding}

This was a collaborative study between Eli Lilly and Company and Guy's and St Thomas' NHS Foundation Trust. The study was sponsored by Eli Lilly and Company. JM and JB were employees of Eli Lilly and Company at the time of the study and contributed to the study design, interpretation of the data and manuscript writing. Medical writing services were provided by Rx Communications, Mold, UK and were funded by Eli Lilly and Company. ICS receives funding from the National Institute for Health Research (NIHR) West Midlands Clinical Research Network (Clinical Trials Scholarship). The views expressed are those of the authors and not necessarily those of the NHS, NIHR or the Department of Health and Social Care.

\section{Availability of data and materials}

The datasets generated and/or analysed during the current study are not publicly available as they represent routinely collected NHS data from a single centre, leading to the potential for patient confidentiality to be compromised.

\section{Ethics approval and consent to participate}

Analysis of data from Guy's RA Centre was approved by the Health Research Authority (IRAS project ID 209418). As the Guy's RA Centre comprises routinely collected anonymised healthcare data, written consent was not required.

\section{Consent for publication}

Not applicable.

\section{Competing interests}

ICS has no competing interests; BK has received support from AbbVie, Arthritis Support UK, Eli Lilly and Company, Janssen, Novartis, F. Hoffmann-La Roche and UCB; JM is an employee of Eli Lilly and Company; JB was an employee of Eli Lilly and Company at the time of the study but is now an employee of Gilead Sciences.

\section{Author details}

${ }^{1}$ Primary Care Centre Versus Arthritis, School of Primary, Community and Social Care, Keele University, Keele, Staffordshire ST5 5BG, UK. ${ }^{2}$ Haywood 
Academic Rheumatology Centre, Haywood Hospital, Midlands Partnership NHS Foundation Trust, High Lane, Burslem, Staffordshire ST6 7AG, UK. ${ }^{3}$ Eli Lilly and Company, Priestly Road, Basingstoke, Hampshire RG24 9NL, UK. ${ }^{4}$ Department of Rheumatology, Guy's and St. Thomas' NHS Foundation Trust, Great Maze Pond, London SE1 9RT, UK.

Received: 26 March 2020 Accepted: 19 August 2020

Published online: 21 October 2020

\section{References}

1. Smolen JS, Breedveld FC, Burmester GR, Bykerk V, Dougados M, Emery P, et al. Treating rheumatoid arthritis to target: 2014 update of the recommendations of an international task force. Ann Rheum Dis. 2016;75(1):3-15.

2. Smolen JS, Landewe R, Bijlsma J, Burmester G, Chatzidionysiou K, Dougados $M$, et al. EULAR recommendations for the management of rheumatoid arthritis with synthetic and biological disease-modifying antirheumatic drugs: 2016 update. Ann Rheum Dis. 2017;76(6):960-77.

3. Singh JA, Saag KG, Bridges SLJ, Akl EA, Bannuru RR, Sullivan MC, et al. 2015 American College of Rheumatology guideline for the treatment of rheumatoid arthritis. Arthritis Care Res (Hoboken). 2016;68(1):1-25.

4. Nikiphorou E, Norton S, Young A, Carpenter L, Dixey J, Walsh DA, et al. Association between rheumatoid arthritis disease activity, progression of functional limitation and long-term risk of orthopaedic surgery: combined analysis of two prospective cohorts supports EULAR treat to target DAS thresholds. Ann Rheum Dis. 2016;75(12):2080-6.

5. Barnabe C, Sun Y, Boire G, Hitchon CA, Haraoui B, Thorne JC, et al. Heterogeneous disease trajectories explain variable radiographic, function and quality of life outcomes in the Canadian early arthritis cohort (CATCH). PLoS One. 2015;10(8):e0135327.

6. Conaghan PG, Hensor EM, Keenan AM, Morgan AW, Emery P, YEAR Consortium. Persistently moderate DAS-28 is not benign: loss of function occurs in early RA despite step-up DMARD therapy. Rheumatology. 2010; 49(10):1894-9.

7. National Institute for Health and Care Excellence (NICE). Rheumatoid arthritis in adults: management (NG100). 2018.

8. Hurkmans E, van der Giesen FJ, Vliet Vlieland TP, Schoones J, Van den Ende ECHM. Dynamic exercise programs (aerobic capacity and/or muscle strength training) in patients with rheumatoid arthritis. Cochrane Database Syst Rev. 2009:4:CD006853.

9. Gullick NJ, Oakley SP, Zain A, Gibson T, Jones T, Mistlin A, et al. Goaldirected therapy for RA in routine practice is associated with improved function in patients with disease duration up to 15 years. Rheumatology (Oxford). 2012;51(4):759-61.

10. Scott IC, Ibrahim F, Panayi G, Cope AP, Garrood T, Vincent A, et al. The frequency of remission and low disease activity in patients with rheumatoid arthritis, and their ability to identify people with low disability and normal quality of life. Semin Arthritis Rheum. 2019;49(1):20-6.

11. Bruce B, Fries JF. The Stanford Health Assessment Questionnaire: dimensions and practical applications. Health Qual Life Outcomes. 2003;1:20.

12. Brooks R. EuroQol: the current state of play. Health Policy (New York). 1996; 37(1):53-72.

13. R Documentation: Package 'bestNormalize'. 20 August 2019. https://cran.rproject.org/web/packages/bestNormalize/bestNormalize.pdf Accessed 20 Aug 2019.

14. Wells GA, Tugwell P, Kraag GR, Baker PR, Groh J, Redelmeier DA. Minimum important difference between patients with rheumatoid arthritis: the patient's perspective. J Rheumatol. 1993;20(3):557-60

15. Allen A, Carville S, McKenna F. Diagnosis and management of rheumatoid arthritis in adults: summary of updated NICE guidance. BMJ. 2018;362:k3015.

16. Norton S, Fu B, Scott DL, Deighton C, Symmons DPM, Wailoo AJ, et al. Health assessment questionnaire disability progression in early rheumatoid arthritis: systematic review and analysis of two inception cohorts. Semin Arthritis Rheum. 2014;44(2):131-44.

17. Pan Y, Norton S, Gwinnutt JM, Kearsley-Fleet L, Symmons DPM, Lunt M, et al. Not all moderate disease is the same - identification of disability trajectories among patients with rheumatoid arthritis and moderate disease activity. PLoS One. 2019;14(5):e0215999.

18. Drossaers-Bakker KW, de Buck M, van Zeben D, Zwinderman AH, Breedveld FC, Hazes JM. Long-term course and outcome of functional capacity in rheumatoid arthritis: the effect of disease activity and radiologic damage over time. Arthritis Rheum. 1999;42(9):1854-60.
19. Combe B, Cantagrel A, Goupille P, Bozonnat M-C, Sibilia J, Eliaou J-F, et al. Predictive factors of 5-year health assessment questionnaire disability in early rheumatoid arthritis. J Rheumatol. 2003;30(11):2344-9.

20. Gullick NJ, Mian AN, Ibrahim F, Walker D, Hassell A, Kiely PDW, et al. Predicting responses in patients with rheumatoid arthritis to diseasemodifying agents using baseline clinical data. Clin Exp Rheumatol. 2017; 35(5):810-5.

21. Fanouriakis A, Papalopoulos I, Gergianaki I, Spyrou G, Erden A, Rapsomaniki P, et al. In early arthritis patients, high HAQ at baseline and DAS28 at three months predict suboptimal outcomes at two years: a retrospective cohort study. Clin Exp Rheumatol. 2018:36(5):806-13.

22. Baganz L, Richter A, Albrecht K, Schneider M, Burmester G-R, Zink A, et al. Are prognostic factors adequately selected to guide treatment decisions in patients with rheumatoid arthritis? A collaborative analysis from three observational cohorts. Semin Arthritis Rheum. 2019;48(6):976-82.

23. Barnett $A G$, van der Pols JC, Dobson AJ. Regression to the mean: what it is and how to deal with it. Int J Epidemiol. 2004:34(1):215-20.

24. Wilcox MA, Chang AMZ, Johnson IR. The effects of parity on birthweight using successive pregnancies. Acta Obstet Gynecol Scand. 1996;75(5):459-63.

25. Kario K, Schwartz JE, Pickering TG. Changes of nocturnal blood pressure dipping status in hypertensives by nighttime dosing of a-adrenergic blocker, doxazosin. Hypertension. 2000;35(3):787-94.

26. Cummings SR, Palermo L, Browner W, Marcus R, Wallace R, Pearson J, et al. Monitoring osteoporosis therapy with bone densitometry. Misleading changes and regression to the mean. JAMA. 2000;283(10):1318-21.

27. Schectman G, Hoffmann RG. A history of hypercholesterolemia influences cholesterol measurements. Arch Intern Med. 1988;148(5):1169-71.

28. Strand V, Wright GC, Bergman MJ, Tambiah J, Taylor PC. Patient expectations and perceptions of goal-setting strategies for disease management in rheumatoid arthritis. J Rheumatol. 2015;42(11):2046-54

29. Heiberg T, Kvien TK. Preferences for improved health examined in 1,024 patients with rheumatoid arthritis: pain has highest priority. Arthritis Rheum. 2002;47(4):391-7.

30. Kavanaugh A, Helliwell P, Ritchlin CT. Psoriatic arthritis and burden of disease: patient perspectives from the population-based multinational assessment of psoriasis and psoriatic arthritis (MAPP) survey. Rheumatol Ther. 2016;3(1):91-102

31. Walsh DA, McWilliams DF. Mechanisms, impact and management of pain in rheumatoid arthritis. Nat Rev Rheumatol. 2014;10:581.

32. Chua JR, Castrejon I, Pincus T. Assessment of pain and other patient symptoms in routine clinical care as quantitative, standardised, "scientific" data. Clin Exp Rheumatol. 2017;35 Suppl 1(5):13-20.

\section{Publisher's Note}

Springer Nature remains neutral with regard to jurisdictional claims in published maps and institutional affiliations.
Ready to submit your research? Choose BMC and benefit from:

- fast, convenient online submission

- thorough peer review by experienced researchers in your field

- rapid publication on acceptance

- support for research data, including large and complex data types

- gold Open Access which fosters wider collaboration and increased citations

- maximum visibility for your research: over $100 \mathrm{M}$ website views per year

At $\mathrm{BMC}$, research is always in progress.

Learn more biomedcentral.com/submissions 